\title{
Does Clot Burden Score on Baseline T2*-MRI Impact Clinical Outcome in Acute Ischemic Stroke Treated with Mechanical Thrombectomy?
}

\author{
Imad Derraz, ${ }^{\text {a }}$ Romain Bourcier, ${ }^{\mathrm{b}}$ Marc Soudant, ${ }^{\mathrm{c}}$ Sébastien Soize, ${ }^{\mathrm{d}}$ Wagih Ben Hassen, ${ }^{\mathrm{e}}$ \\ Gabriella Hossu, ${ }^{\mathrm{f}}$ Frederic Clarencon, ${ }^{\mathrm{g}}$ Anne Laure Derelle, ${ }^{\mathrm{a}}$ Marie Tisserand, ${ }^{\mathrm{h}}$ Helene Raoult, ${ }^{\mathrm{i}}$ \\ Laurence Legrand, ${ }^{\mathrm{e}}$ Serge Bracard, ${ }^{\mathrm{a}}$ Catherine Oppenheim, ${ }^{\mathrm{e}}$ Olivier Naggara, ${ }^{\mathrm{e}}$ on behalf of the \\ THRACE Investigators \\ aDepartment of Neuroradiology, CHRU Nancy, INSERM, University of Lorraine, Nancy, France \\ bepartment of Neuroradiology, CHU Nantes, Nantes, France \\ 'Department of Biostatistics, CHRU Nancy, INSERM, University of Lorraine, Nancy, France \\ 'Department of Neuroradiology, CHU Reims, Reims, France \\ eParis Descartes University, INSERM UMR 894 and Department of Neuroradiology, Sainte-Anne Hospital Center, Paris, France \\ fDepartment of CIC1433 Innovative Technology, CHRU Nancy, INSERM, University of Lorraine, Nancy, France \\ ${ }^{9}$ Department of Neuroradiology, Pitié-Salpêtrière Hospital, Paris, France \\ hDepartment of Neuroradiology, Foch Hospital, Suresnes, France \\ 'Department of Neuroradiology, CHU Rennes, Rennes, France
}

Background and Purpose A long clot, defined by a low (0-6) clot burden score (CBS) assessed by T2* MR sequence, is associated with worse clinical outcome after intravenous thrombolysis (IVT) for acute ischemic stroke than is a small clot (CBS, 7-10). The added benefit of mechanical thrombectomy (MT) might be higher in patients with long clot. The aim of this pre-specified post hoc analysis of the THRombectomie des Artères CErebrales (THRACE) trial was to assess the association between T2*$\mathrm{CBS}$, successful recanalization and clinical outcome.

Methods Of 414 patients randomized in the THRACE trial, 281 patients were included in this analysis. Associations between T2*-CBS and clinical outcome on the modified Rankin Scale (mRS) at 3 months were tested.

Results High T2*-CBS, i.e., small clot, was associated with a shift toward better outcome on the mRS; proportional odds ratio (POR) per point CBS was 1.19 (95\% confidence interval [Cl], 1.05 to 1.34) in the whole population, $1.34(95 \% \mathrm{Cl}, 1.13$ to 1.59$)$ in IVT group, and $1.04(95 \% \mathrm{Cl}, 0.87$ to 1.23$)$ in IVTMT group. After adjustment for baseline prognostic variables, the effect of the full scale T2*-CBS was not statistically significant in the whole population and for the IVTMT group but remains significant for the IVT group (POR, 1.32; 95\% Cl, 1.11 to 1.58).

Conclusions A small clot, as assessed using $\mathrm{T}^{*}$-CBS, is associated with improved outcome and may be used as a prognostic marker. Despite the worst outcome with long clot, the relative benefit of MT over IVT seemed to increase with low T2*-CBS and longer clot.
Correspondence: Olivier Naggara Paris Descartes University, INSERM UMR 894 and Department of Neuroradiology, Sainte-Anne Hospital Center, 1 rue Cabanis, 75014 Paris, France

Tel: $+33-1-4565-8574$

Fax: $+33-1-4565-8470$

E-mail: o.naggara@ch-sainte-anne.fr

Received: July 4, 2018

Revised: September 8, 2018

Accepted: October 2, 2018

KeyWords Ischemic stroke; Magnetic resonance imaging; Thrombosis; Endovascular recanalization 


\section{Introduction}

Recanalization of the arterial occlusion is the cornerstone of treatment in acute ischemic stroke (AIS) patients. Several randomized clinical trials (RCTs) ${ }^{1-7}$ and an individual patient data meta-analysis ${ }^{8}$ have recently shown that mechanical thrombectomy (MT) combined with standard treatment (including intravenous thrombolysis [IVT]) was superior to standard treatment alone, with higher rates of reperfusion, more frequent favorable functional outcome, and lower 3 months mortality, for severe AIS caused by large-vessel occlusion (LVO) in the anterior circulation. The cumulative evidence from these studies resulted in the modification of practice guidelines and profound changes in worldwide stroke care organization. ${ }^{9}$

Despite its compelling efficacy, up to half of AIS-LVO patients do not regain functional independence after MT. ${ }^{8}$ This unfavorable outcome is largely attributable to unsuccessful mechanical recanalization. ${ }^{1-4,7}$ Amongst factors influencing recanalization success, clot length is an important determinant, ${ }^{10-12}$ that was scarcely assessed in the recent RCTs. Indeed, if patients were included in case of LVO on computed tomography angiography (CTA), only few studies ${ }^{13,14}$ assessed clot length using the CTA-defined clot burden score (CTA-CBS). These analyses demonstrated a direct link between clot length, likelihood of recanalization, final infarct volumes, and 3-month neurological outcome.

Brain magnetic resonance imaging (MRI), using the ${ }^{*}{ }^{*}-\mathrm{MRI}$ sequence, is a powerful tool to identify thrombus in AIS patients, based on the presence of a susceptibility vessel sign (SVS)..$^{15,16}$ Clot length is included in the $\mathrm{T}^{*}-\mathrm{CBS}_{1}{ }^{17}$ in which a lower score reflects longer thrombus. $2^{*}$-CBS was recently used after IVT or MT, as a predictor of recanalization and functional outcome. ${ }^{17,18}$ However, as for CTA-CBS, ${ }^{19}$ these studies were mainly retrospective and no adjustment was performed for crucial baseline prognostic variables such as initial National Institutes of Health Stroke Scale (NIHSS) score or recanalization results.

With more than 300 patients included with pre-treatment MRI, the THRombectomie des Artères CErebrales (THRACE) trial (ClinicalTrials.gov, number NCT01062698) offers a unique opportunity to study the associations between $\mathrm{T}^{*}$-CBS, successful recanalization rate and functional independence. The purpose of this prespecified post hoc analysis of the THRACE trial was to determine the relation between $\mathrm{T} 2^{*}$-CBS and the effect on endovascular treatment and neurological improvement in AIS-LVO patients.

\section{Methods}

\section{Study design}

THRACE was a randomized controlled trial done in 26 centers in France. Study design and protocol have been previously detailed. ${ }^{7}$ AIS-LVO patients were randomly assigned in a 1:1 ratio to receive either IVT alone (IVT group) or IVT+MT (IVTMT group). IVT had to be started within 4 hours and MT within 5 hours of symptom onset. Occlusions had to be confirmed by CTA or magnetic resonance angiography (MRA). Before randomization, written informed consent was obtained from all patients or their legal representatives. The study protocol was approved by
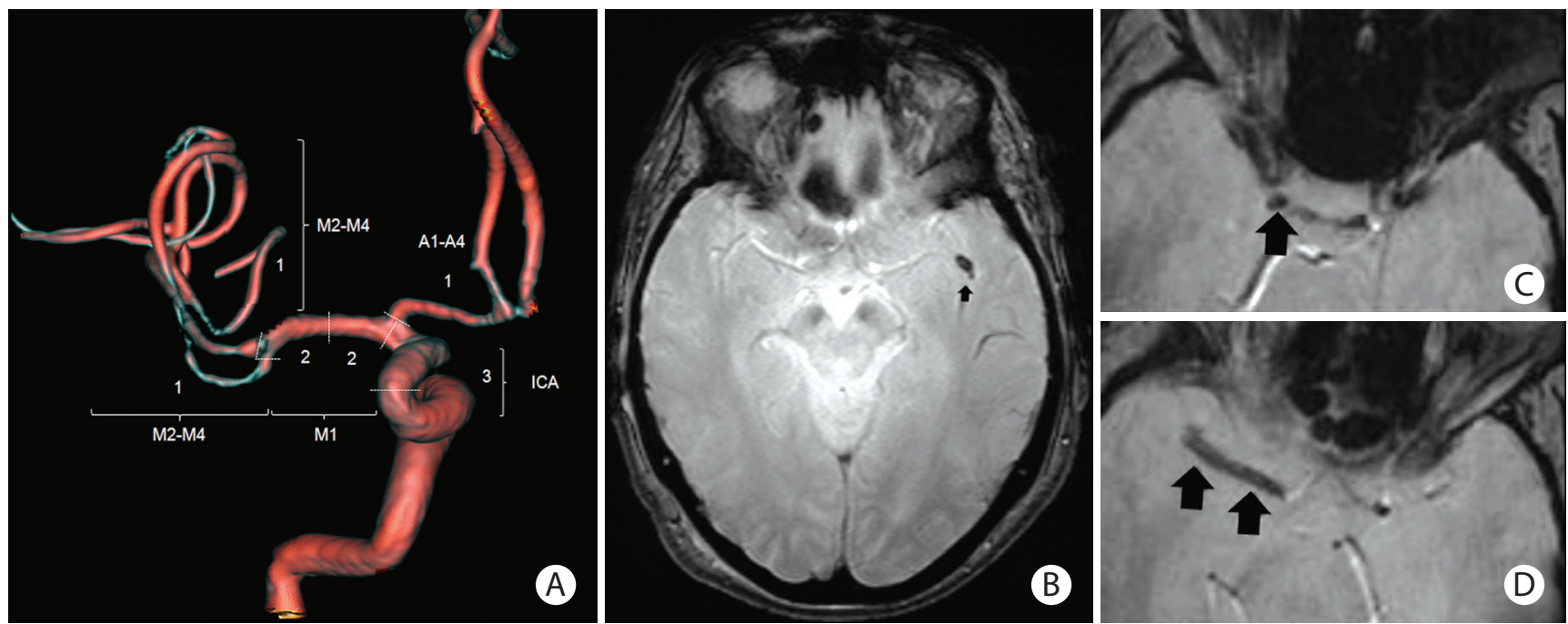

Figure 1. The T2*-clot burden score (T2*-CBS). (A) A score of 10 is normal, implying absence of susceptibility vessel sign (SVS) on T2* Three points (as indicated) are subtracted for SVS found in the supraclinoid internal carotid artery (ICA), 2 points for SVS in each of the proximal and distal halves of the middle cerebral artery trunk (M1), 1 point for SVS in the A1-A4 segment and 2 points for SVS in the M2-M4 branches. A score of 0 implying complete multisegment vessel occlusion. (B) Patient 1, distal occlusion with T2*-CBS=9 (SVS in 1 left M2 branch). (C, D) Patient 2, proximal occlusion with T2*-CBS=3 (SVS in right supraclinoid ICA, proximal and distal halves of M1). 
the Comité de Protection des Personnes (CPP) III Nord Est Ethics Committee and the research boards of the participating centers. No data were reported on thrombus characteristics obtained on admission brain MRI.

\section{Outcomes measures}

The primary outcome in the THRACE trial was the proportion of patients with a score of 0-2 on the modified Rankin Scale (mRS), indicating functional independence, at 3 months after the intervention. Secondary outcomes nonexhaustively included, successful recanalization, defined as a modified Thrombolysis in Cerebral Infarction (mTICl) score $\geq 2 b^{20}$ in the IVTMT group, and the NIHSS score at 24 hours in all patients. Clinical assessments were done by vascular neurologists who were not masked to the treatment to which the patients had been allocated.

\section{Image analysis}

MRI images and angiograms before and after MT were reviewed by four experienced neuroradiologists, who were masked to randomization group and patient clinical outcome. Baseline examinations included the determination of the Alberta Stroke Program Early CT Score (ASPECTS) ${ }^{21}$ on diffusion weighted imaging (DWI) sequence and the location of the arterial occlusion by MRA. After initial training, two experienced observers from the core imaging committee searched for SVS, that is, a hypointense signal on $\mathrm{T}^{*}$ within a vascular cistern, exceeding the size of the homologous contralateral arterial diameter. If present, an appropriate $\mathrm{T} 2^{*}$-CBS was assigned according to the methods of Legrand et al. ${ }^{17} \mathrm{~T} 2^{*}$-CBS is a 10-point scoring system used to define the extent of thrombus in the anterior circulation (Figure 1). Because susceptibility artifact at the skull base prevents evaluation of the infraclinoid internal carotid artery (ICA), this segment was not analyzed and 3 points were assigned to the supraclinoid ICA level, for consistency with the CTA-CBS. A score of 10 implies clot absence. A score of 0 implies complete multisegment vessel occlusion by a long clot. T2*-CBS was subsequently dichotomized using a >6-point cut-off (0-6: long clot vs. 7-10: small clot), according to and for comparison with CTA-CBS studies. ${ }^{13}$

\section{Statistical analysis}

All primary outcome analyses were performed according to the intention-to-treat principle. For this study, the primary effect variable was the proportional adjusted common odds ratio for a shift in the direction of better outcome on the 3-month mRS. The association between full-scale or dichotomized T2*-CBS (0-6 vs. 7-10) with shift in the direction of better outcome on the mRS was assessed using ordinal logistic regression respectively.

For all outcome parameters, two models were generated as previously used..$^{13}$ Model A contained the T2*-CBS variable and treatment. In model $B$, the main prognostic baseline variables were added: age, stroke severity (NIHSS score), glycemia, and ASPECTS score. An interaction term of treatment allocation with $2^{*}$-CBS was added to the both unadjusted and adjusted models to assess whether $\mathrm{T} 2{ }^{*}$-CBS was a treatment effect modifier. The models with and without added interaction term (nested models) were compared using the chi-square test.

Patient characteristics are reported for groups with and without SVS and patient with long (T2*-CBS 0-6) and small clot (T2*-CBS 7-10). Continuous variables were compared with Student t-test, a Mann-Whitney test, or Median test, as appropriate. Categorical variables were compared using chisquare or Fisher exact test, as appropriate.

For all statistical analyses, $P<0.05$ was considered statistically significant. All statistical analyses were done with SAS/ STAT version 9.4 (SAS Institute Inc., Cary, NC, USA).

\section{Results}

\section{Patient characteristics}

Among 414 patients randomized in the THRACE trial (Figure 2), initial imaging work-up was performed using MRI in 153 of 208 patients (74\%) in the IVT group and 148 of 204 patients $(73 \%)$ in the IVTMT group ( $P=0.83)$. Of these, 20 patients were excluded leaving 281 patients for analysis in the present study.

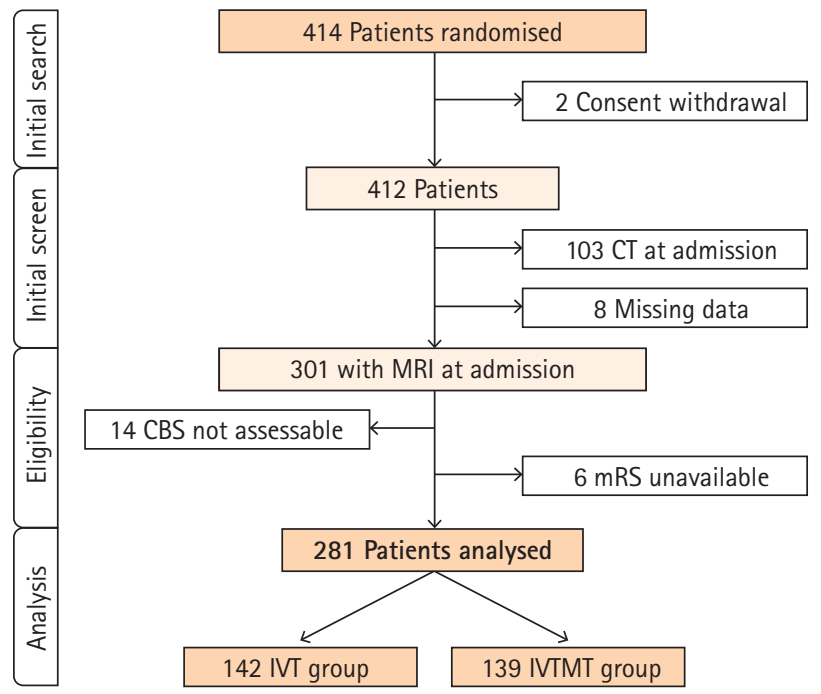

Figure 2. Flow chart study. CT, computerized tomography; MRI, magnetic resonance imaging; CBS indicates clot burden score; mRS, modified Rankin Scale; IVT, intravenous thrombolysis; IVTMT, intravenous thrombolysis mechanical thrombectomy. 
Table 1. Baseline patient characteristics according to dichotomized T2*-CBS groups

\begin{tabular}{|c|c|c|c|}
\hline Characteristic & $\mathrm{T} 2^{*}-\mathrm{CBS}(7-10)$ & $\mathrm{T} 2^{*}-\mathrm{CBS}(0-6)$ & $P$ \\
\hline Number & 170 & 111 & \\
\hline IVTMT randomization group & $87(51.2)$ & $52(46.8)$ & $0.48^{*}$ \\
\hline Undergo IVTMT & $57(33.5)$ & $43(38.7)$ & $0.37^{*}$ \\
\hline Age (yr) & $61.8 \pm 14.2$ & $65.4 \pm 13.4$ & $0.01^{+}$ \\
\hline$\leq 70$ & $109(64.1)$ & $50(45.0)$ & $0.002^{*}$ \\
\hline$>70$ & 61 (35.9) & $61(55.0)$ & \\
\hline Sex & & & $0.32^{*}$ \\
\hline Male & $93(54.7)$ & $54(48.6)$ & \\
\hline Female & $77(45.3)$ & $57(51.4)$ & \\
\hline \multicolumn{4}{|l|}{ Comorbidities } \\
\hline Hypertension & $84(49.7)$ & $57(52.3)$ & $0.67^{*}$ \\
\hline Diabetes mellitus & $20(11.8)$ & $5(4.6)$ & $0.04^{*}$ \\
\hline History of stroke & $9(5.5)$ & $9(8.3)$ & $0.37^{*}$ \\
\hline Hypercholesterolemia & $79(53.0)$ & $50(50.5)$ & $0.70^{*}$ \\
\hline Current tobacco use & $43(28.3)$ & $14(14.1)$ & $0.009^{*}$ \\
\hline Coronary disease & $20(12.4)$ & $20(18.7)$ & $0.16^{*}$ \\
\hline Etiology of cerebral infarction & & & $0.41^{*}$ \\
\hline Large-artery atherosclerosis & $25(15.3)$ & $13(13.0)$ & \\
\hline Cardioembolism & 64 (39.3) & $51(51.0)$ & \\
\hline Small-vessel occlusion & $1(0.6)$ & $0(0)$ & \\
\hline Other determined etiology & $10(6.1)$ & $5(5.0)$ & \\
\hline Undetermined etiology & 63 (38.7) & $31(31.0)$ & \\
\hline Baseline NIHSS score & $18(13-21)$ & $17(14-20)$ & $0.69^{\ddagger}$ \\
\hline ASPECTS at baseline & $7(5-9)$ & $7(4-8)$ & $0.72^{+}$ \\
\hline ASPECTS (0-4) & $25(14.7)$ & $28(25.2)$ & $0.04^{*}$ \\
\hline ASPECTS (5-7) & $73(42.9)$ & $35(31.5)$ & \\
\hline ASPECTS (8-10) & $72(42.4)$ & $48(43.2)$ & \\
\hline Baseline occlusion location (\%) & & & $<0.001^{\S}$ \\
\hline ICA & $13(7.6)$ & $30(27.0)$ & \\
\hline M1 & $155(91.2)$ & $81(73.0)$ & \\
\hline M2 & $2(1.2)$ & $0(0)$ & \\
\hline \multicolumn{4}{|l|}{ Workflow time (min) } \\
\hline From stroke onset to imaging & $112(88-135)$ & 112 (89-142) & $0.91^{+}$ \\
\hline From stroke onset to IVT & $146(124-170)$ & $154(120-180)$ & $0.49^{*}$ \\
\hline From stroke onset to recanalization & $239(208-270)$ & 261 (204-291) & $0.44^{+}$ \\
\hline ASPECTS at 24 hours & $7(4-8)$ & $6(3-8)$ & $0.40^{+}$ \\
\hline
\end{tabular}

Values are presented as number (\%), mean \pm standard deviation, or median (interquartile range).

CBS, clot burden score; IVTMT, intravenous thrombolysis mechanical thrombectomy; NIHSS, National Institutes of Health Stroke Scale; ASPECTS, Alberta Stroke Program Early CT Score; ICA, internal carotid artery; IVT, intravenous thrombolysis.

${ }^{*}$ Chi-square test; ${ }^{+}$Mann-Whitney test; ${ }^{*}$ Median test; ${ }^{\S}$ Fisher exact test.

Baseline characteristics are summarized in Supplementary Table 1. Sociodemographic characteristics, comorbidities, and baseline NIHSS did not differ between IVT and IVTMT groups, nor delays from symptom onset to IVT initiation or to randomization.

\section{Baseline characteristics according to $2^{*}$-CBS group}

Among 281 patients included in 19 centers, 234 (83.3\%) demonstrated presence of SVS, $118(50.4 \%)$ in the IVT group, and $116(49.6 \%)$ in the IVTMT group. Overall, the median T2*- 
Table 2. Patient characteristics of dichotomized mRS groups

\begin{tabular}{|c|c|c|c|}
\hline Characteristic & $\mathrm{mRS} \leq 2$ & $m R S>2$ & $P$ \\
\hline Number & 144 & 137 & \\
\hline IVTMT randomization group & 79 (54.9) & $60(43.8)$ & $0.06^{*}$ \\
\hline Age $(y r)$ & $60.1 \pm 14.6$ & $66.5 \pm 12.7$ & $<0.001^{+}$ \\
\hline$\leq 70$ & $97(67.4)$ & $62(45.3)$ & $<0.001^{*}$ \\
\hline$>70$ & $47(32.6)$ & $75(54.7)$ & \\
\hline Sex & & & $0.94^{*}$ \\
\hline Male & $75(52.1)$ & $72(52.6)$ & \\
\hline Female & $69(47.9)$ & $65(47.4)$ & \\
\hline \multicolumn{4}{|l|}{ Comorbidities } \\
\hline Hypertension & $61(43.0)$ & $80(58.8)$ & $0.01^{*}$ \\
\hline Diabetes mellitus & $10(7.0)$ & $15(11.1)$ & $0.23^{*}$ \\
\hline History of stroke & $11(7.8)$ & $7(5.3)$ & $0.41^{*}$ \\
\hline Hypercholesterolemia & $68(52.3)$ & $61(51.7)$ & $0.92^{*}$ \\
\hline Current tobacco use & $30(21.7)$ & $27(23.9)$ & $0.69^{*}$ \\
\hline Coronary disease & 16 (11.4) & $24(18.8)$ & $0.09^{*}$ \\
\hline Baseline NIHSS score & $16(12-19)$ & $19(16-22)$ & $<0.001^{\ddagger}$ \\
\hline ASPECTS at baseline & $7(6-9)$ & $6(4-8)$ & $<0.01^{*}$ \\
\hline ASPECTS (0-4) & $16(11.1)$ & $37(27.0)$ & $0.002^{*}$ \\
\hline ASPECTS (5-7) & $58(40.3)$ & $50(36.5)$ & \\
\hline ASPECTS (8-10) & 70 (48.6) & $50(36.5)$ & \\
\hline Baseline occlusion location (\%) & & & $<0.001^{\S}$ \\
\hline ICA & $10(6.9)$ & $33(24.1)$ & \\
\hline M1 & $133(92.4)$ & 103 (75.2) & \\
\hline M2 & $1(0.7)$ & $1(0.7)$ & \\
\hline \multicolumn{4}{|l|}{ Workflow time (min) } \\
\hline From stroke onset to imaging & $111(85-140)$ & $114(93-137)$ & $0.80^{\ddagger}$ \\
\hline From stroke onset to IVT & $146(120-179)$ & $150(125-175)$ & $0.53^{+}$ \\
\hline From stroke onset to recanalization & $240(204-280)$ & $250(208-281)$ & $0.57^{\ddagger}$ \\
\hline SVS (+) & $120(83.3)$ & $114(83.2)$ & $0.98^{*}$ \\
\hline SVS length & $14.3(10.6-18.7)$ & $17.4(12.9-23.6)$ & $<0.04^{\dagger}$ \\
\hline $\mathrm{T} 2^{*}-\mathrm{CBS}>6$ & $97(67.4)$ & $73(53.3)$ & $0.02^{*}$ \\
\hline Recanalizers ( $\mathrm{TICl} \geq 2 \mathrm{~b})$ & 43 & 25 & $<0.001^{*}$ \\
\hline ASPECTS at 24 hours & $8(6-9)$ & $5(2-7)$ & $<0.001^{*}$ \\
\hline NIHSS score at 24 hours & $5(2-9)$ & 18 (14-22) & $<0.001^{\dagger}$ \\
\hline
\end{tabular}

Values are presented as number (\%), mean \pm standard deviation, or median (interquartile range).

mRS, modified Rankin Scale; IVTMT, intravenous thrombolysis mechanical thrombectomy; NIHSS, National Institutes of Health Stroke Scale; ASPECTS, Alberta Stroke Program Early CT Score; ICA, internal carotid artery; IVT, intravenous thrombolysis; SVS, susceptibility vessel sign; CBS, clot burden score; TICl, Thrombolysis in Cerebral Infarction.

${ }^{*}$ Chi-square test; ${ }^{+}$Mann-Whitney test; ${ }^{\dagger}$ Median test; ${ }^{\circledR}$ Fisher exact test.

CBS was 7 (interquartile range, 6 to 8), similar in IVT and IVTMT groups $(P=0.38)$. After dichotomizing the T2*-CBS, 111 patients $(39.5 \%)$ had a long clot and $170(60.5 \%)$ a small clot. Patients with a long clot were significantly older, had a lower DWI-ASPECTS score, more often had diabetes mellitus or were active smokers at baseline (Table 1).

\section{Primary outcome}

The primary outcome was assessed in 281 patients (Table 2). At 3 months, 79 of 139 patients (56.8\%) in the IVTMT group and 65 of $142(45.8 \%)$ in the IVT group had functional independence. The primary outcome was not influenced by the presence of a SVS (120/144 in mRS 0-2 and 114/137 in mRS >2, $P=0.98$ ). 
Table 3. Estimates of POR of T2*-CBS using ordinal logistic regressions explaining lower modified Rankin Scale score at 3 months

\begin{tabular}{lccccccc}
\hline \multirow{2}{*}{ Proportional odds ratio for } & \multicolumn{3}{c}{ POR $^{+}$} & \multicolumn{3}{c}{ APOR $^{*}$} \\
\cline { 2 - 3 } & IVT subgroup & IVTMT subgroup & All & & IVT subgroup & IVTMT subgroup & All \\
\hline Increase of 1 point of CBS & 1.34 & 1.04 & 1.19 & 1.32 & 0.94 & 1.12 \\
& $(1.13-1.59)^{*}$ & $(0.87-1.23)$ & $(1.05-1.34)^{*}$ & $(1.11-1.58)^{*}$ & $(0.79-1.12)$ & $(0.99-1.27)$ \\
CBS $>6$ vs. CBS $\leq 6$ & 3.19 & 0.93 & 1.73 & 3.06 & 0.71 & 1.48 \\
& $(1.75-5.82)^{*}$ & $(0.51-1.7)$ & $(1.13-2.65)^{*}$ & $(1.63-5.74)^{*}$ & $(0.38-1.32)$ & $(0.95-2.30)$ \\
\hline
\end{tabular}

Adjustment on age, National Institutes of Health Stroke Scale (NIHSS), glycemia, and Alberta Stroke Program Early CT Score (ASPECTS).

POR, proportional odds ratio; CBS, clot burden score; APOR, adjusted proportional odds ratio; IVT, intravenous thrombolysis; IVTMT, intravenous thrombolysis mechanical thrombectomy.

*Statistically significant ( $P \leq 0.05) ;{ }^{+} \mathrm{POR}$ of lower mRS for T2*-CBS (with 95\% confidence interval) estimated by Model A, i.e., without adjustment; ${ }^{\ddagger} \mathrm{APOR}$ of lower mRS for T2*-CBS (with 95\% confidence interval) estimated by Model B.
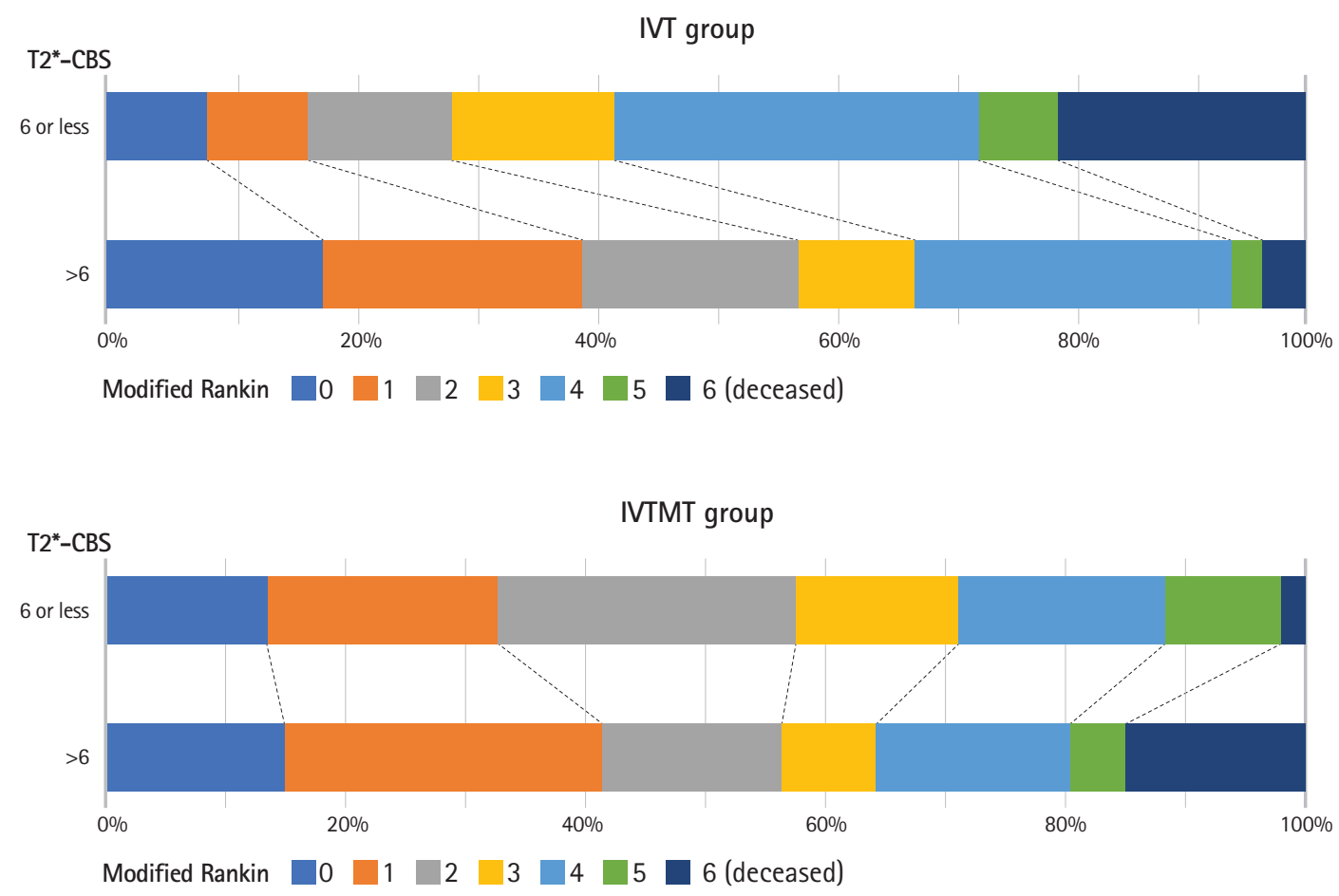

Figure 3. Modified Rankin Scale distribution for the intra-arterial treatment and control arms for T2*-CBS groups (0-6) and (7-10). CBS, clot burden score; IVT, intravenous thrombolysis; and IVTMT, intravenous thrombolysis mechanical thrombectomy.

Proportion of small clot was significantly higher in patients with favorable (97/144 patients, 67.4\%) than in patients with unfavorable outcome $(73 / 137,53.3 \% ; 0 R, 1.81 ; 95 \%$ confidence interval $[\mathrm{Cl}], 1.12$ to $2.94 ; P=0.016)$. Small clot was associated with a shift toward better outcome on the mRS; proportional odds ratio (POR) per each T2*-CBS point was, with randomization group adjustment: $1.19(95 \% \mathrm{Cl}, 1.05$ to 1.34$)$, $1.34(95 \% \mathrm{Cl}, 1.13$ to 1.59$)$, and 1.04 (0.87 to 1.23 ) (interaction, $P=0.03$ ) in the whole population, in IVT and IVTMT groups, respectively. After adjustment, the effect of the full scale T2* CBS was not statistically significant in the whole population (POR, 1.12; $95 \% \mathrm{Cl}, 0.99$ to $1.27 ; P=0.1$ ) and for the IVTMT group (POR, $0.94 ; 95 \% \mathrm{Cl}, 0.79$ to $1.12 ; P=0.73$ ) but remains significant for the IVT group (POR, 1.32; 95\% $\mathrm{Cl}, 1.11$ to 1.58). Considering the dichotomized $\mathrm{T}^{*}$-CBS, similar results were found (Table 3 and Figure 3).

\section{Secondary outcomes}

Successful recanalization was observed in 66 of 91 patients (72.5\%) in the IVTMT group who received MT treatment. There was no association between the presence of SVS and recanalization result $(P=0.45)$ nor influence of the $\mathrm{T} 2^{*}-\mathrm{CBS}$, considered as a continuous variable ( $P=0.75)$ or dichotomized ( $7-10$ vs. $\leq 6$, $P=0.57$ ) (Supplementary Table 2). 


\section{Discussion}

In this prespecified post hoc analysis of THRACE trial, longer clot as assessed using $T 2^{*}$-CBS was associated with a higher likelihood of unfavorable neurological outcome. In analysis of 3-month ordinal mRS, adjusted on randomization group, there was a 19\% relative increase in the likelihood of a worse outcome with every point decrease in $\mathrm{T} 2^{*}$-CBS. Despite the worst outcome with long clot, the relative benefit of IVTMT over IVT seemed to increase with longer thrombus.

We found a significant interaction between treatment allocation and clot length as measured using $\mathrm{T}^{*}$-CBS. In the recent $\mathrm{RCTs}_{1}{ }^{1-7}$ evaluation of intracranial thrombus burden was performed on CTA. In the ESCAPE trial, Puetz et al. ${ }^{22}$ demonstrated an increase in benefit from MT for lower CTA-CBS, i.e., longer thrombus, when compared with higher CTA-CBS. Similarly, in a post hoc analysis of 108 patients included in the THERAPY trial, ${ }_{1}^{14}$ longer thrombi, as defined on CTA, were independently associated with worse clinical outcomes. Furthermore, in adjusted analyses of 90day ordinal mRS, there was a 33\% relative increase in the likelihood of a worse outcome with every 5-mm increase in thrombus length and the relative benefit of MT compared with IVT alone increased with thrombus length. ${ }^{14}$ Our results stand in apparent contradiction with the post hoc analysis of the MR CLEAN trial, which did not establish thrombus length as a treatment effect modifier. ${ }^{13}$ In this latter study, the underestimation of the increased benefit of MT over IVT in longer thrombi can be tentatively explained by the fact that CTA may overestimate the extent of thrombus involvement. Indeed, if the collateral circulation is weak or with short delays between contrast injection and imaging acquisition, ${ }_{1}^{23}$ an overestimation of clot length is possible. In addition, although CTA has been demonstrated to provide accurate thrombus length measurement, ${ }^{24}$ it is less sensitive than MR susceptibility weighted sequences. ${ }^{25}$ However, there are no comparative studies of clot length imaged with both CT and MRI.

The greater beneficial effect in IVTMT group accounts for the paradoxical finding of increasing relative benefit of MT despite the overall worse outcome associated with longer thrombi. This finding illustrates the difference between prognostic and therapeutic imaging biomarkers; in AIS-LVO patients, long clot is simultaneously a negative prognostic biomarker and a positive therapeutic biomarker with regards to MT (i.e., clot length modifies the differential treatment effect of MT or IVT). The detrimental effect of low CBS on clinical outcome is most likely attributable to greater difficulty of recanalizing longer and/or multisegment thrombi. ${ }^{14,24}$ Other explanation of the worst outcomes seen in lower T2*-CBS may be that longer clot were associated with lower baseline ASPECTS. In case of longer clot, there is a higher probability of occlusion of the lenticulostriate and insula perforators, for which collateral compensation is limited. In addition, an inverse correlation between pial collaterals strength and clot length has been demonstrated. ${ }^{14}$ Weaker collaterals may contribute to both the extent of the thrombus and a reduction in cerebral blood flow to the ischemic penumbra, potentiating the extent and degree of injury, hence contributing to worse clinical outcomes. Taken together, worst outcome seen with long clot is likely the consequence of a synergistic effect of poor collateral strength, larger baseline infarct and longer thrombotic occlusion that is difficult to rapidly recanalize.

In line with THERAPY trial results, ${ }^{14}$ we did not demonstrate any influence of the $T 2^{*}$-CBS on recanalization result, in the IVTMT group. As a difference, in our study, patients were not included based on clot length whereas in THERAPY trial, only patients harboring thrombi $>8 \mathrm{~mm}$ were included. A non-randomized study of mostly stent-retriever (SR) MT also demonstrated no relationship between thrombus length, as measured on SVS, and successful recanalization. ${ }^{26}$ Our study reinforces the idea that, if longer thrombi are unlikely to recanalize in IVT patients, length did not impact efficacy of MT.

Recently, randomized comparison of first-line MT with ADAPT technique versus SR did not result in an increased successful revascularization rate. ${ }^{27}$ However, in the ASTER trial, thrombus length was short with mean values of 13 and $11.5 \mathrm{~mm}$, in ADAPT and SR groups, respectively. Furthermore, in a recent post hoc analysis of the ASTER trial, the first-line MT strategy (aspiration vs. stent) did not result in an increased successful reperfusion rate in AIS-LVO patients according to the admission CBS. ${ }^{28}$ Identifying the best MT method to address LVOs with long clot, i.e., T2*-CBS (0-6), will most likely be a challenge, notably because of the expected limited added benefit of a device association choice versus another, resulting in anticipated power issues. Knowledge of other thrombus characteristics, such as complex composition, tensile, compressive, rheological, and frictional properties, which might contribute to their relative resistance to clot removal during MT, may help in optimizing endovascular devices and strategies. ${ }^{29,30}$ Until more data are available, clot length, assessed using $\mathrm{T}^{*}$-CBS, is a reasonable approach to guide treatment strategies and select patients in future trials.

The strength of our study is that, beyond the demonstration of a LVO, no other imaging criteria was used to select patients in the THRACE trial. Hence, patients with unfavorable clinical and imaging profiles were included, resulting in generalizable findings. The present study was based on the largest to date AIS-LVO population initially included based on brain MRI data in a RCT and allows for a less biased assessment of the efficacy of baseline imaging prognostic biomarkers to select patients 
for future studies.

A few points may require clarification. First, 59 patients randomized to the intervention arm did not receive MT. When evaluating imaging biomarker of efficacy for MT, it could add to the sensitivity of the analysis to only include the patients who actually received MT. A second limitation is that T2*-CBS likely underestimates full clot extent, given that the susceptibility effect depends on thrombus composition and age..$^{15}$ An additional limitation is the variability in MRI measurement. ${ }^{31}$ Indeed, the extent of the SVS blooming artifact might vary with different magnetic field strengths and echo time. Future studies should examine the impact of imaging acquisition parameters on the T2*-CBS variability. Finally, 47 of 281 patients did not demonstrate SVS and were included in the analysis as $\mathrm{T} 2^{*}-\mathrm{CBS}=10$. This may have underestimated the real clot length in these patients.

\section{Conclusions}

Clot length, as assessed using the MRI based T2*-CBS is independently associated with functional outcome in patients with AIS caused by a LVO, and may be used as prognostic biomarker. Despite the worst outcome with long clot, the relative benefit of MT over IVT seemed to increase with low $2^{*}$-CBS.

\section{Supplementary materials}

Supplementary materials related to this article can be found online at https://doi.org/10.5853/jos.2018.01921.

\section{Disclosure}

The authors have no financial conflicts of interest.

\section{Acknowledgments}

The funder of the study (French Ministry for Health) had no role in study design, data collection, data analysis, data interpretation, or writing of the report. The corresponding author had full access to all the data in the study and had final responsibility for the decision to submit for publication.

\section{Appendix}

THRACE investigators: Alain Bonafé (Department of Neuroradiology, Gui de Chauliac Hospital, Montpellier, France), Xavier Leclerc (Department of Radiology, University Hospital of Lille, Lille, France), Nelly Agrinier (Department of Clinical Epidemiology INSERM CIC-EC 1433, University of Lorraine and University
Hospital of Nancy, Nancy, France), Serge Bakchine (Department of Neurology, University Hospital of Reims, Reims, France), Flore Baronnet (Stroke Unit, Pitié-Salpêtrière Hospital Group and Paris 6 University-Pierre et Marie Curie, Paris, France), Marine Beaumont (Department of INSERM CIC-IT, University of Lorraine and University Hospital of Nancy, Nancy, France), Yannick Bejot (Department of Neurology, University Hospital of Dijon, Dijon, France), Jerome Berge (Department of Interventional and Diagnostic Neuroradiology, University Hospital of Bordeaux, Bordeaux, France), Marc Bintner (Department of Neuroradiology Sud-Reunion Hospital Group, Saint Pierre, France), Romain Bourcier (Department of Interventional and Diagnostic Neuroradiology, University Hospital of Nantes, Nantes, France), Tae Hee Cho (Department of Neurology, University Hospital of Lyon, Lyon, France), Frédéric Clarencon (Department of Interventional Neuroradiology Pitié-Salpêtrière Hospital Group and Paris 6 University-Pierre et Marie Curie, Paris, France), Julien Cogez (Department of Neurology, University Hospital of Caen, Caen, France), Charlotte Cordonnier (Department of Neurology, University Hospital of Lille, Lille, France), Christian Denier (Department of Neurology, University Hospital of Bicêtre, Le Kremlin-Bicêtre, France), Anne Laure Derelle (Department of Diagnostic and Interventional Neuroradiology, University Hospital of Nancy, Nancy, France), Olivier Detante (Department of Neurology, University Hospital of Grenoble, Grenoble, France), Anthony Faivre (Department of Neurology, Hôpital d'Instruction des Armées, Sainte Anne, Toulon, France), Anne Ferrier, (Department of Neurology, University Hospital Gabriel-Montpied, Clermont-Ferrand, France), Laetitia Gimenez (Department of Neurology, University Hospital of Limoges, Limoges, France), Sophie Godard (Department of Neurology, University Hospital of Angers, Angers, France), Gregoire Boulouis (Department of Neuroradiology, Sainte-Anne Hospital and Paris- Descartes University, INSERM U894, Paris, France), Benoit Guillon (Department of Neurology, University Hospital of Nantes, Nantes, France), Emmanuel Houdart (Department of Neuroradiology, University Hospital Lariboisière, Paris, France), Bertrand Lapergue (Department of Neurology, Foch Hospital, Suresnes, France), Mariano Musacchio (Department of Neuroradiology, Pasteur Hospital, Colmar, France), Olivier Naggara (Department of Neuroradiology, Sainte-Anne Hospital and Paris-Descartes University, INSERM U894, Paris, France), Jean Philippe Neau (Department of Neurology, University Hospital of Poitiers, Poitiers, France), Michael Obadia (Department of Neurology, Rothschild Ophthalmological Foundation, Paris, France), Anne Pasco-Papon (Department of Radiology, University Hospital of Angers, Angers, France), Michel Piotin (Department of Interventional Neuroradiology [MP] Rothschild Ophthalmological Foundation, Paris, 
France), Laurent Pierot (Department of Neuroradiology, University Hospital of Reims, Reims, France), Helene Raoult (Department of Neuroradiology, University Hospital of Rennes, Rennes, France), Sébastien Richard (Department of Neurology University Hospital of Nancy, Nancy, France), Frederic Ricolfi (Department of Neuroradiology, University Hospital of Dijon, Dijon, France), Thomas Ronziere (Department of Neurology, University Hospital of Rennes, Rennes, France), Guillaume Saliou (Department of Neuroradiology, University Hospital of Bicêtre, Le Kremlin-Bicêtre, France), Igor Sibon (Department of Neurology, University Hospital of Bordeaux, Bordeaux, France), Sebastien Soize (Department of Neuroradiology, University Hospital of Reims, Reims, France), Jacques Sedat (Department of Radiology, University Hospital of Nice, Nice, France), Christian Stapf (Department of Neurology, University Hospital Lariboisière, Paris, France), Laurent Suissa (Department of Neurology, University Hospital of Nice, Nice, France), Marie Tisserand (Department of Neuroradiology, Sainte-Anne Hospital and Paris- Descartes University, INSERM U894, Paris, France), Francis Turjman (Department of Interventional Neuroradiology, University Hospital of Lyon, Lyon, France), and Stephane Velasco (Departments of Radiology, University Hospital of Poitiers, Poitiers, France).

\section{References}

1. Berkhemer OA, Fransen PS, Beumer D, van den Berg LA, Lingsma $\mathrm{HF}_{1}$, Yoo $\mathrm{AJ}$, et al. $A$ randomized trial of intraarterial treatment for acute ischemic stroke. N Eng/ J Med 2015;372:11-20.

2. Goyal M, Demchuk AM, Menon BK, Eesa M, Rempel JL, Thornton $\mathrm{J}$, et al. Randomized assessment of rapid endovascular treatment of ischemic stroke. N EnglJ Med 2015;372:1019-2030.

3. Campbell BC, Mitchell PJ, Kleinig TJ, Dewey HM, Churilov L, Yassi $\mathrm{N}$, et al. Endovascular therapy for ischemic stroke with perfusion-imaging selection. N EnglJ Med 2015;372:1009-1018.

4. Saver JL, Goyal M, Bonafe A, Diener HC, Levy El, Pereira VM, et al. Stent-retriever thrombectomy after intravenous t-PA vs. t-PA alone in stroke. N Engl J Med 2015;372:2285-2295.

5. Jovin TG, Chamorro A, Cobo E, de Miquel MA, Molina CA, Rovira $A$, et al. Thrombectomy within 8 hours after symptom onset in ischemic stroke. N Engl J Med 2015;372:2296-2306.

6. Mocco J, Zaidat 00, von Kummer R, Yoo AJ, Gupta R, Lopes D, et al. Aspiration thrombectomy after intravenous alteplase versus intravenous alteplase alone. Stroke 2016;47:2331-2338.

7. Bracard S, Ducrocq $X$, Mas JL, Soudant M, Oppenheim C, Moulin $T$, et al. Mechanical thrombectomy after intravenous alteplase versus alteplase alone after stroke (THRACE): a randomised controlled trial. Lancet Neurol 2016;15:1138-1147.

8. Goyal M, Menon BK, van Zwam WH, Dippel DW, Mitchell PJ,
Demchuk AM, et al. Endovascular thrombectomy after largevessel ischaemic stroke: a meta-analysis of individual patient data from five randomised trials. Lancet 2016;387:1723-1731.

9. Powers WJ, Derdeyn CP, Biller J, Coffey CS, Hoh BL, Jauch EC, et al. 2015 American Heart Association/American Stroke Association focused update of the 2013 guidelines for the early management of patients with acute ischemic stroke regarding endovascular treatment: a guideline for healthcare professionals from the American Heart Association/American Stroke Association. Stroke 2015;46:3020-3035.

10. Tan IY, Demchuk AM, Hopyan J, Zhang L, Gladstone D, Wong $\mathrm{K}$, et al. CT angiography clot burden score and collateral score: correlation with clinical and radiologic outcomes in acute middle cerebral artery infarct. AJNR Am J Neuroradiol 2009;30:525-531.

11. Yan S, Chen $\mathrm{Q}, \mathrm{Xu}$ M, Sun J, Liebeskind DS, Lou M. Thrombus length estimation on delayed gadolinium-enhanced $\mathrm{T} 1$. Stroke 2016;47:756-761.

12. Sillanpaa N, Saarinen JT, Rusanen $H$, Hakomaki J, Lahteela $A$,

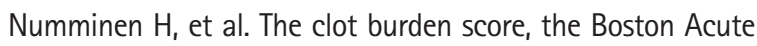
Stroke Imaging Scale, the cerebral blood volume ASPECTS, and two novel imaging parameters in the prediction of clinical outcome of ischemic stroke patients receiving intravenous thrombolytic therapy. Neuroradiology 2012;54:663-672.

13. Treurniet KM, Yoo AJ, Berkhemer OA, Lingsma HF, Boers AM, Fransen PS, et al. Clot burden score on baseline computerized tomographic angiography and intra-arterial treatment effect in acute ischemic stroke. Stroke 2016;47:2972-2978.

14. Yoo AJ, Khatri P, Mocco J, Zaidat OO, Gupta R, Frei D, et al. Impact of thrombus length on outcomes after intra-arterial aspiration thrombectomy in the THERAPY trial. Stroke 2017;48: 1895-1900.

15. Flacke $S$, Urbach $H$, Keller $E$, Träber $F$, Hartmann $A$, Textor J, et al. Middle cerebral artery (MCA) susceptibility sign at susceptibility-based perfusion MR imaging: clinical importance and comparison with hyperdense MCA sign at CT. Radiology 2000;215:476-482.

16. Naggara 0 , Raymond J, Domingo Ayllon M, Al-Shareef $F_{\text {, }}$ Touzé $E_{1}$ Chenoufi $M$, et al. T2* "susceptibility vessel sign" demonstrates clot location and length in acute ischemic stroke. PLoS One 2013;8:e76727.

17. Legrand L, Naggara O, Turc G, Mellerio C, Roca P, Calvet D, et al. Clot burden score on admission T2*-MRI predicts recanalization in acute stroke. Stroke 2013;44:1878-1884.

18. Soize S, Batista AL, Rodriguez Regent C, Trystram D, Tisserand $M$, Turc $G$, et al. Susceptibility vessel sign on $T 2^{*}$ magnetic resonance imaging and recanalization results of mechanical thrombectomy with stent retrievers: a multicentre 
cohort study. Eur J Neuro/ 2015;22:967-972.

19. Heo JH, Kim K, Yoo J, Kim YD, Nam HS, Kim EY. Computed tomography-based thrombus imaging for the prediction of recanalization after reperfusion therapy in stroke. J Stroke 2017;19:40-49.

20. Zaidat 00, Yoo AJ, Khatri P, Tomsick TA, von Kummer R, Saver $J \mathrm{~L}$, et al. Recommendations on angiographic revascularization grading standards for acute ischemic stroke: a consensus statement. Stroke 2013;44:2650-2663.

21. Barber PA, Demchuk AM, Zhang J, Buchan AM. Validity and reliability of a quantitative computed tomography score in predicting outcome of hyperacute stroke before thrombolytic therapy. ASPECTS Study Group. Alberta Stroke Programme Early CT Score. Lancet 2000;355:1670-1674.

22. Puetz V, Barlinn K, Bodechtel U, Campbell B, Linn J, Gerber JC. Imaging-based selection for revascularization in acute ischemic stroke. Curr Opin Neurol 2016;29:20-29.

23. Frölich AM, Schrader D, Klotz E, Schramm R, Wasser $K_{1}$ Knauth $M$, et al. 4D CT angiography more closely defines intracranial thrombus burden than single-phase CT angiography. AJNR Am J Neuroradiol 2013;34:1908-1913.

24. Riedel $\mathrm{CH}$, Jensen $U$, Rohr $A$, Tietke $M$, Alfke $K$, Ulmer $S$, et al. Assessment of thrombus in acute middle cerebral artery occlusion using thin-slice nonenhanced computed tomography reconstructions. Stroke 2010;41:1659-1664.

25. Gratz PP, Schroth G, Gralla J, Mattle HP, Fischer U, Jung S, et al. Whole-brain susceptibility-weighted thrombus imaging in stroke: fragmented thrombi predict worse outcome. AJNR
Am J Neuroradiol 2015;36:1277-1282.

26. Weisstanner C, Gratz PP, Schroth G, Verma RK, Köchl A, Jung S, et al. Thrombus imaging in acute stroke: correlation of thrombus length on susceptibility-weighted imaging with endovascular reperfusion success. Eur Radiol 2014;24:1735-1741.

27. Lapergue B, Blanc R, Gory B, Labreuche J, Duhamel A, Marnat $G$, et al. Effect of endovascular contact aspiration vs stent retriever on revascularization in patients with acute ischemic stroke and large vessel occlusion: the ASTER randomized clinical trial. JAMA 2017;318:443-452.

28. Zhu F, Lapergue $B$, Kyheng $M$, Blanc $R$, Labreuche J, Ben Machaa $M$, et al. Similar outcomes for contact aspiration and stent retriever use according to the admission clot burden score in ASTER. Stroke 2018;49:1669-1677.

29. Bourcier R, Brecheteau N, Costalat V, Daumas-Duport B,

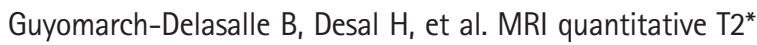
mapping on thrombus to predict recanalization after endovascular treatment for acute anterior ischemic stroke. J Neuroradiol 2017;44:241-246.

30. Bourcier R, Mazighi M, Labreuche J, Fahed R, Blanc R, Gory $B$, et al. Susceptibility vessel sign in the ASTER trial: higher recanalization rate and more favourable clinical outcome after first line stent retriever compared to contact aspiration. $J$ Stroke 2018;20:268-276.

31. Bourcier R, Détraz L, Serfaty JM, Delasalle BG, Mirza M, Derraz I, et al. MRI interscanner agreement of the association between the susceptibility vessel sign and histologic composition of thrombi. J Neuroimaging 2017;27:577-582. 
Supplementary Table 1. Baseline characteristics of stroke patients with intravenous thrombolysis alone or intravenous thrombolysis plus mechanical thrombectomy

\begin{tabular}{|c|c|c|c|}
\hline Sociodemographic characteristic & IVT alone ( $n=142)$ & IVTMT (n=139) & $P$ \\
\hline Age (yr) & $62.8 \pm 15.2$ & $63.7 \pm 12.7$ & $0.59^{*}$ \\
\hline$\leq 70$ & $79(55.6)$ & $80(57.6)$ & $0.75^{+}$ \\
\hline$>70$ & $63(44.4)$ & $59(42.4)$ & \\
\hline Sex & & & $0.08^{+}$ \\
\hline Male & $67(47.2)$ & $80(57.6)$ & \\
\hline Female & 75 (52.8) & $59(42.4)$ & \\
\hline \multicolumn{4}{|l|}{ Comorbidities } \\
\hline Hypertension & $77(54.2)$ & $64(47.1)$ & $0.23^{+}$ \\
\hline Diabetes mellitus & $17(12.0)$ & $8(5.9)$ & $0.08^{+}$ \\
\hline History of stroke & $9(6.4)$ & $9(6.8)$ & $0.89^{+}$ \\
\hline Hypercholesterolemia & $71(55.0)$ & $58(48.7)$ & $0.32^{+}$ \\
\hline Current or past tobacco use & $52(40.3)$ & $55(44.7)$ & $0.48^{+}$ \\
\hline Coronary disease & $20(14.7)$ & $20(15.2)$ & $0.92^{+}$ \\
\hline Baseline NIHSS score & $18(14-21)$ & $18(14-20)$ & $0.99^{\ddagger}$ \\
\hline ASPECTS at baseline & $7(5-9)$ & $7(6-8)$ & $0.12^{+}$ \\
\hline \multicolumn{4}{|l|}{ Workflow time (min) } \\
\hline From stroke onset to MR imaging & $110(90-138)$ & $114(84-138)$ & $0.57^{+}$ \\
\hline From stroke onset to IVT & $149(124-180)$ & $150(120-175)$ & $0.94^{+}$ \\
\hline From stroke onset to randomization & 167 (137-197) & 163 (142-192) & $0.474^{\ddagger}$ \\
\hline Baseline occlusion location (\%) & & & $0.047^{\S}$ \\
\hline ICA & $28(19.7)$ & $15(10.8)$ & \\
\hline M1 & $114(80.3)$ & $124(89.2)$ & \\
\hline
\end{tabular}

Values are presented as mean \pm standard deviation, number (\%), or median (interquartile range).

IVT, intravenous thrombolysis; IVTMT, intravenous thrombolysis mechanical thrombectomy; NIHSS, National Institutes of Health Stroke Scale; ASPECTS, Alberta Stroke Program Early CT Score; MR, magnetic resonance; ICA, internal carotid artery.

${ }^{*}$ Student t-test; ${ }^{+}$Chi-square test; ${ }^{\dagger}$ Median test; ${ }^{\text {F }}$ Fisher exact test. 
Supplementary Table 2. Comparison between recanalizers $(\mathrm{TICl} \geq 2 \mathrm{~b})$ and non-recanalizers for demographic, clinical and imaging data

\begin{tabular}{|c|c|c|c|}
\hline Characteristic & $m R S \leq 2$ & $m R S>2$ & $P$ \\
\hline Number & 24 & 66 & \\
\hline Age (yr) & $64.9 \pm 12.4$ & $62.0 \pm 13.7$ & $0.33^{*}$ \\
\hline$\leq 70$ & $14(58.3)$ & $38(57.6)$ & $0.95^{+}$ \\
\hline$>70$ & $10(41.7)$ & $28(42.4)$ & \\
\hline Sex & & & $0.08^{+}$ \\
\hline Male & $17(70.8)$ & $33(50.0)$ & \\
\hline Female & $7(29.2)$ & $33(50.0)$ & \\
\hline \multicolumn{4}{|l|}{ Comorbidities } \\
\hline Hypertension & $13(54.2)$ & $25(39.1)$ & $0.20^{+}$ \\
\hline Diabetes mellitus & $1(4.2)$ & $2(3.1)$ & $1^{+}$ \\
\hline History of stroke & $1(4.2)$ & $3(4.8)$ & $1^{+}$ \\
\hline Hypercholesterolemia & $8(42.1)$ & $25(41.7)$ & $0.97^{+}$ \\
\hline Current tobacco use & $3(15.0)$ & 17 (28.8) & $0.22^{+}$ \\
\hline Coronary disease & $1(4.3)$ & $7(11.1)$ & $0.68^{*}$ \\
\hline Baseline NIHSS score & $19(16-21)$ & $18(15-21)$ & $0.34^{\S}$ \\
\hline ASPECTS at baseline & $6(4-8)$ & $7(6-8)$ & $0.39^{\S}$ \\
\hline ASPECTS at 24 hours & $4(2-6)$ & $7(5-8)$ & $0.002^{\mathrm{s}}$ \\
\hline ASPECTS (0-4) & $14(60.9)$ & $12(18.2)$ & $<0.001^{\dagger}$ \\
\hline ASPECTS (5-7) & $7(30.4)$ & $26(39.4)$ & \\
\hline ASPECTS (8-10) & $2(8.7)$ & $28(42.4)$ & \\
\hline Baseline occlusion location (\%) & & & $0.154^{\dagger}$ \\
\hline ICA & $5(20.8)$ & $6(9.1)$ & \\
\hline M1 & 19 (79.2) & $60(90.9)$ & \\
\hline \multicolumn{4}{|l|}{ Workflow time (min) } \\
\hline From stroke onset to imaging & 110 (94-129) & $108(85-135)$ & $0.85^{\S}$ \\
\hline From stroke onset to IVT & 148 (134-170) & $138(117-170)$ & $0.34^{\S}$ \\
\hline From stroke onset to recanalization & $250(230-270)$ & $234(200-279)$ & $0.29^{\S}$ \\
\hline SVS (+) & $20(83.3)$ & $60(90.9)$ & $0.45^{+}$ \\
\hline $\mathrm{T} 2{ }^{*}-\mathrm{CBS}>6$ & $14(58.3)$ & $34(51.5)$ & $0.57^{+}$ \\
\hline NIHSS score at 24 hours & $18(13-22)$ & $6(4-14)$ & $<0.001^{\S}$ \\
\hline
\end{tabular}

Values are presented as mean \pm standard deviation, number $(\%)$, or median (interquartile range).

TICl, Thrombolysis in Cerebral Infarction; mRs, modified Rankin Scale; NIHSS, National Institutes of Health Stroke Scale; ASPECTS, Alberta Stroke Program Early CT Score; ICA, internal carotid artery; IVT, intravenous thrombolysis; SVS, susceptibility vessel sign; CBS, clot burden score.

${ }^{*}$ Mann-Whitney test; ${ }^{+}$Chi-square test; ${ }^{\dagger}$ Fisher exact test; ${ }^{\circledR}$ Median test. 